\title{
Seguimiento serológico de infección por Trypanosoma cruzi en individuos estudiados de 50 municipios del Estado de Jalisco, México de 1987 a 1994
}

\author{
Serological follow-up of Trypanosoma cruzi infection from 1987 \\ to 1994 in 50 counties of the State of Jalisco, Mexico
}

\author{
Francisco Trujillo Contreras, Maria de los Angeles Villanueva Yerenas, \\ Margarita Soto Gutiérrez, Miguel Raygoza Anaya y Alberto Jiménez Corder ${ }^{1}$
}

\begin{abstract}
Resumen La Universidad de Guadalajara decidió realizar en 1987 un estudio seroepidemiológico para conocer la prevalencia de la infección por Trypanosoma cruzi en los 124 municipios del Estado de Jalisco, México, en el cual se obtuvieron tasas de 21,6 por 100 habitantes. De diciembre de 1993 a junio de 1994 se realizó un seguimiento de algunos de esos individuos, estudiándose sólo 2669 de ellos, de 50 municipios de áreas rurales del estado. Las causas de no encuesta de los faltantes fueron diversas, estando entre las más importantes la emigración $(27,6)$ y la renuencia $(14,8)$. Encontramos a 476 positivos $(17,8 \%)$ y 2193 negativos $(82,2 \%)$. Este segundo estudio nos permitió detectar 80 infectados nuevos (2,9\%) lo que representa una tasa de incidencia de 30 por 1000 habitantes. Sin embargo, encontramos una seroconversión en los individuos estudiados, ya que de 1007 individuos que estaban positivos en 1987, 943 de ellos continúan así, mientras, que 64 individuos se negativizaron (2,3\%), no siendo esto imputable a fallas de laboratorio, la prevalencia de positividad detectada en ambos estudios fue casi idéntica 18,1 y $17,8 \%$ respectivamente, por lo que nosotros pensamos que las condiciones en que viven los individuos de las localidades rurales del Estado de Jalisco favorecen a que las infecciones continúen.
\end{abstract}

Palabras-claves: Enfermedad de Chagas. Trypanosoma cruzi. Seguimiento serológico. Jalisco, México.

\begin{abstract}
In 1987 the University of Guadalajara performed a seroepidemiological survey in order to know the prevalence of infection for Trypanosoma cruzi in the 124 counties of the state of Jalisco, Mexico in which appraisals of 21.6 for 100 inhabitants were gotten. From December of 1993 to June of 1994 was carried out a pursuit of some those individuals, studying it only to 2669 of they, of 50 municipalities of rural areas of the state. The causes off not survey of the not found was diverse, being between the most important the emigration $(27.6 \%)$ and the not cooperation (14.8\%). We found to 476 positives (17.8\%) and 2193 negatives (82.2\%). This second study allowed us detect 80 new cases (2.9\%) the one which represents an appraisal of incidence of 30 for 100 inhabitants. However we found a seroconvertion in the studied individuals, since of 1007 individuals that were positives in 1987; 943 of them continues so, while 64 individuals became negative (2.3\%), not being this attributable to flaws of laboratory, the prevalence of positivite detected in both studies was almost identical, 18.1 and $17.8 \%$ respectly, for the one which we believe that the conditions in that the individuals of the rural towns of the state of Jalisco live favor to that the infections continue.
\end{abstract}

Key-words: Chagas' disease. Serology. Trypanosoma cruzi infection. Jalisco, Mexico.

\footnotetext{
1. División de Disciplinas Básicas. Departamentos de Patología y de Salud Pública, Universidad de Guadalajara, Jalisco, México. Dirección para correspondencia: Dr. Francisco Trujillo Contreras. Carlos Mérida 348. Colinas de la Normal, Casilla Postal 44270, Guadalajara, Jalisco, México.

Tel: (3) 824-1080.

e-mail: ftc@cusur.udg.mx

Recebido para publicação em 17/8/99.
} 
La enfermedad de Chagas es causada por un protozoario hemoflagelado el cual produce diferentes manifestaciones clínicas en humanos. Muchos pacientes continúan en una forma indeterminada y asintomáticos. Algunos de estos pacientes pueden desarrollar lesiones 10 ó 20 años después de la infección, 20 a $30 \%$ presentan la forma cardiaca de la enfermedad ${ }^{8}$ y alrededor del $7 \%$ tiene megaesófago ${ }^{1}$. La prevalencia de pacientes con megacolon no está muy clara aún.

Esta enfermedad ha sido estudiada en forma amplia en Brasil y Argentina, sin embargo, en nuestro país no existen todavía centros de investigación, como el Oswaldo Cruz o el Mario Fatala Chaben, que nos permitan realizar estudios tan amplios que incluyan la serología, por lo que nosotros intentamos estudiar el proceso infeccioso de esta manera.

En México, Mazzoti ${ }^{7}$ logra descubrir los dos primeros casos humanos en 1940 buscando diagnosticar paludismo. En el Estado de Jalisco Cuartero y Ponce ${ }^{2}$ publicaron en 1967 los dos primeros casos de esta enfermedad encontrados en el municipio de Santa María de los Angeles. Posteriormente, en el año de 1986 se descubre en el municipio de Tuxcueca, Jalisco el brote epidémico de enfermedad de Chagas aguda más importante de los reportados para esta enfermedad, en donde 11 individuos, de una familia de 14, inician con la sintomatología ${ }^{3}$. Estos sucesos hicieron que el Estado de Jalisco ocupara el primer lugar de casos agudos y crónicos reportados en la República Mexicana.

Ante estos acontecimientos, la Universidad de Guadalajara decidió en 1987 realizar un estudio seroepidemiológico para conocer la prevalencia de la infección por Trypanosoma cruzien los 124 municipios del Estado de Jalisco, obteniendo una tasa de 21,6 por 100 habitantes $^{10}$.

Sin embargo, a pesar de la tasa de infección tan elevada que se encontró, de 1940 a 1994 en México sólo se han reportado, aproximadamente, 300 casos agudos y 300 casos crónicos comprobados de enfermedad $^{9}$, (el gran número de casos de Jalisco son sólo de infectados), lo cual nos llama mucho la atención, ya que en nuestro país, tenemos un medio ambiente en el área rural que no difiere mucho de Brasil y Argentina, las viviendas de los campesinos son similares, tenemos también especies de reduvidos capaces de transmitir la enfermedad (incluso más especies que en países sudamericanos); y el agente etiológico lo hemos encontrado prácticamente en todos los mamíferos de sangre caliente, pero, no tenemos la magnitud de casos clínicos que se tienen en esos países.

Por esta razón, se decidió realizar un seguimiento serológico de infección por Trypanosoma cruzi a los individuos que se estudiaron en 1987, para ver qué cambios sucedieron en el transcurso de 7 años, proponiendo una hipótesis de negación donde esperamos no encontrar cambios en los individuos con serología positiva y negativa.

\section{MATERIAL Y MÉTODOS}

El estudio comprende dos fases y se trata de una investigación híbrida, compuesta por un estudio de antes-después y un estudio de incidencia.

Primera fase, 1987. El estudio partió de una investigación prévia, la cual se llevó a cabo en 1987 y fue un proyecto de tipo observacional, descriptivo y transversal en donde la unidad muestreal fue la familia y su vivienda y, se llevó a cabo en los 124 municipios del Estado de Jalisco. Se determinó un tamaño de muestra y se seleccionaron al azar los individuos a estudiar, la información se registró en una cédula de encuesta familiar y se tomó por venopunción muestras para la realización de las pruebas serológicas.

Segunda fase, 1994. Es una investigación de incidencia y de diseño no experimental, de antes-después o pre y post-evaluatorio. Es un diseño híbrido porque mezcla dos modelos de investigación, es de incidencia porque parte de los casos conocidos en 1987 y es de antesdespués porque se tiene una medición inicial (serología y características de la vivienda) que permite examinar cambios a través del tiempo con relación al mejoramiento de la vivienda. En estos estudios hay intervención, la cual se consideró como los cambios realizados a la vivienda por sus habitantes.

Durante 1994, se tomaron muestras de sangre periférica a los habitantes de los 50 municipios. Se excluyeron del estudio a los nacidos de 1987 en adelante y a los individuos que vivían en la misma casa habitación, pero, que no se estudiaron anteriormente. Se revisaron las características de las viviendas para comparar si hubo o no mejoría, se hizo búsqueda de triatomas intra y peridomiciliariamente identificándose género y especie y presencia o ausencia de T. cruzi en ellos. 
Las muestras se extrajeron por venopunción, centrifugándose a 2500 revoluciones por 15 minutos y el suero fue trasladado en refrigeración al Centro de Investigación de Enfermedades Tropicales de la Universidad de Guadalajara. Se realizaron alícuotas y se guardaron congeladas a -20 grados centígrados hasta el momento de realizar las pruebas.

Para su procesamiento se utilizaron las técnicas de hemaglutinación indirecta con un procedimiento similar al realizado por el Instituto Mario Fatala Chaben de Argentina ${ }^{4}$, pero, respetando la técnica marcada por el antígeno comercial Chaga-test de laboratorios Wiener ${ }^{11}$; y la técnica de Inmunofluorescencia Indirecta. Se utilizó nuevamente la cepa Tulawen para no tener variación de antígeno en la prueba. Los títulos que se consideraron positivos, en ambas pruebas, fueron de 1:64 hacia arriba.

El universo de trabajo estuvo constituido por los 5535 individuos que fueron investigados en 1987 y que correspondían a los 50 municipios que se estudiaron (se enlistan a seguir), pero, de ellos y por causas que comentaremos, sólo se pudieron localizar a 2669 que fue nuestra población en estudio. En cada una de las viviendas visitadas se hizo una búsqueda y captura de insectos reduvidos, una revisión de las características de la vivienda para compararla de cómo se encontraba en 1987 y se aplicó una cédula de encuesta familiar para obtener otras variables.
Listado de municipios estudiados en el Estado de Jalisco, Mexico.
1 Arenal

2 Atenguillo

3 Chapala

4 Cuquio

5 Degollado

6 El Salto

7 Etzatlan

8 Guadalajara

9 Ixtlahua. De Membrillo

10 Jalostotitlan

11 Jamay

13 Jocotepec

14 Lagos de Moreno

15 La Huerta

16 Magdalena

17 Mazamitla

18 Mezquitic

19 Ocotlan

20 Ojuelos

21 Poncitlan

22 Puerto Vallarta

23 San Cristobal

24 San Juan De Loslagos

25 San Julian
12 Jesus Maria
26 San Martín De Bolaños

27 San Martín Hidalgo

28 San Miguel El Alto

29 Santa Maria de Los Angeles

30 Sayula

31 Tapalpa

32 Techaluta

33 Tenamaxtlan

34 Tepatitlan

35 Tequila

36 Teuchitlan

37 Tizapan El Alto

38 Tlajomulco

39 Tlaquepaque

40 Toliman

41 Tonala

42 Totatiche

43 Tototlan

44 Tuxcueca

45 Valle de Juárez

46 Villa Corona

47 Villa Purificación

48 Zapopan

49 Zapotlanejo

50 Zapotiltic
La recolección de la información se realizó en cédulas de encuesta, para capturarse posteriormente en un procesador de textos Word Perfect y en una base de datos Dbase 3.1 y las pruebas estadísticas a través del sistema Epi info 6 y de SPSS-PC.

\section{RESULTADOS}

En 1987 en estos 50 municipios se estudiaron un total de 5535 individuos de los cuales sólo se logró tomar nuevamente muestra serológica a 2669 que corresponde al 48,2\% con relación al estudio anterior. De los individuos estudiados encontramos 476 positivos $(17,8 \%$ ) y 2193 negativos $(82,2 \%)$, variando en forma mínima la tasa de seropositividad de 1987, que fue de $18,1 \%$ para estos 50 municipios. Sin embargo, llama mucho la atención una serie de diferencias serológicas encontradas, ya que de los 1007 individuos que resultaron positivos en 1987, 64 de ellos $(2,3 \%)$ tuvieron una seroconversión de positivos a negativos, 80 se encontraron como casos nuevos $(2.9 \%)$ y sólo se pudieron localizar a 396 individuos (39,9\%) de los positivos de 87 (Tabla 1).

Las causas de no estudio de los faltantes fueron: migración, 1530 individuos $(27,6 \%)$, renuencia $817(14,7 \%)$, defunción $188(3,4 \%)$, y otras causas $331(5,9 \%)$ (Tabla 2$)$.

Tabla 1 - Prevalencia de infección de individuos estudiados en 1987 y 1994.

\begin{tabular}{lcccc}
\hline & & 1987 & & \multicolumn{1}{c}{1994} \\
\cline { 2 - 4 } & número & tasa & número & tasa \\
\hline Estudiados & 5.535 & 100,0 & 2.669 & 476 \\
Positivos & 1.007 & 18,2 & 17,8 & 2.193 \\
Negativos & 4.528 & 81,8 & 82,2 & 2.0 \\
\hline
\end{tabular}

tasa $\times 100$ habitantes;

Fuente: cédulas de encuesta seroepidemiológica. 
Tabla 2 - Distribución porcentual de causa de no encuesta en individuos estudiados en 1987.

\begin{tabular}{lcc}
\hline Causas & Número & Porcentaje \\
\hline Fallecidos & 188 & 3,4 \\
Emigrados & 1530 & 27,6 \\
Renuentes & 817 & 14,7 \\
No conocidos & 331 & 5,9 \\
\hline Sub-total & 2866 & 51,8 \\
\hline Estudiados & 2669 & 48,2 \\
\hline Total & 5535 & 100,0 \\
\hline
\end{tabular}

Fuente: cedulas de encuesta seroepidemiologica.

Análisis de asociación. Se consideró la vivienda con riesgo (variable independiente) como aquella vivienda que no tuvo mejoría completa, es decir si, dentro de la vivienda persistía un área de riesgo para facilitar el nacimiento y crecimiento de los triatomas, como puede ser el techo, las paredes o el piso, al relacionar está variable con la serología presentada por los individuos se detectó un OR de $1,1(\mathrm{IC}=0,9-1,4)$ y una $\mathrm{p}>0,05$ (Tabla 3).

Tabla 3 - Asociacion entre serologia detectada en individuos en 1994 y caracteristicas de la vivienda.

\begin{tabular}{|c|c|c|c|c|c|c|}
\hline \multirow[b]{3}{*}{ Vivienda } & \multicolumn{4}{|c|}{ Serologia } & \multirow{3}{*}{ OR } & \multirow{3}{*}{ I.C. $95 \%$} \\
\hline & \multicolumn{2}{|c|}{ positiva } & \multicolumn{2}{|c|}{ negativa } & & \\
\hline & $\mathrm{n}^{\circ}$ & $\%$ & $\mathrm{n}^{\circ}$ & $\%$ & & \\
\hline Con mejoria & 185 & 6,9 & 925 & 34,6 & 1,0 & \\
\hline Sin mejoria & 291 & 10,9 & 1268 & 47,5 & 1,15 & $(0,9-1,4)$ \\
\hline Total & 476 & 17,8 & 2193 & 82,1 & & \\
\hline
\end{tabular}

$\mathrm{p}=0,20107$

Fuente: cedulas de encuesta seroepidemiologica.

Sin embargo, al cruzar la presencia del vector con la serología, encontramos que 270 individuos sí estuvieron con la presencia del vector y 206 no, el valor de p es de $<0,05$ con un OR 1,9 (IC $=1,5-2,3)$ y los límites de confianza no incluyen el 1, por lo que se concluye que no existe significancia estadística entre estas dos variables (Tabla 4).

$\mathrm{Al}$ analizar las viviendas con riesgo contra los casos nuevos encontramos que hubo 21 casos nuevos que sí mejoraron la vivienda y 59 casos que no lo hicieron lo que da un valor de $p<0,004$ y un OR de 2,0 $(I C=1,2-3,5)$ con límites de confianza que no incluyen el 1. La misma situación se da con la presencia del vector como factor de riesgo contra casos nuevos, ya que muestra un valor de $\mathrm{p}<0.0000002$ y un OR de $3,4(\mathrm{IC}=2,1-5,8)$ con límites de confianza que no incluyen el 1 (Tabla 5).

La tasa de incidencia encontrada en el estudio fue de 30 por 1000 habitantes.

Tabla 4 - Asociación entre serologia y presencia del vector en la vivienda en individuos estudiados en 1994.

\begin{tabular}{|c|c|c|c|c|c|c|}
\hline \multirow[b]{3}{*}{ Vector } & \multicolumn{4}{|c|}{ Serologia } & \multirow{3}{*}{ OR } & \multirow{3}{*}{ I.C. $95 \%$} \\
\hline & \multicolumn{2}{|c|}{ positiva } & \multicolumn{2}{|c|}{ negativa } & & \\
\hline & $\mathrm{n}^{\circ}$ & $\%$ & $\mathrm{n}^{\circ}$ & $\%$ & & \\
\hline No & 206 & 7,7 & 1307 & 49,0 & 1,0 & \\
\hline $\mathrm{Si}$ & 270 & 10,1 & 886 & 33,2 & 1,9 & $(1,5-2,3)$ \\
\hline Total & 476 & 17,8 & 2193 & 82,2 & & \\
\hline
\end{tabular}

$p=0.00003$

Fuente: cédulas de encuesta seroepidemiológica. 
Tabla 5 - Asociación entre presentación de casos nuevos y mejoria de las viviendas en individuos estudiados en 1994.

\begin{tabular}{lcccccc}
\hline & \multicolumn{3}{c}{ Casos nuevos } & & \multirow{2}{*}{ OR } & I.C. 95\% \\
\cline { 2 - 7 } Vivienda & \multicolumn{2}{c}{ positiva } & negativa & & \\
\hline Con mejoria & 21 & 0,9 & 925 & 40,7 & 1,0 & \\
Sin mejoria & 59 & 2,6 & 1268 & 55,8 & 2,0 & $(1,2-3,5)$ \\
\hline Total & 80 & 3,5 & 2193 & 96,5 & & \\
\hline
\end{tabular}

$p=0.00645$

Fuente: cédulas de encuesta seroepidemiológica.

\section{DISCUSIÓN}

Al analizar los resultados de las pruebas serológicas, nos tranquiliza que, el porcentaje de positivos no se haya modificado casi en nada, porque a pesar de que sólo estudiamos la mitad de individuos, la prevalencia fue más o menos similar, (18,1 para 87 y 17,8 para 94$)$, lo que nos habla de que las condiciones socioeconómicas en nuestro país en el área rural no se han modificado grandemente en el transcurso de 7 años.

El análisis de los positivos y los negativos encontrados en este estudio de 1994 y su relación con la mejoría de la vivienda no nos muestra que exista una relación significativa o un odds ratio alto, ya que los porcentajes coinciden grandemente, pero, al realizar el análisis con la presencia del vector observamos que sí existe una significancia estadística y un odds ratio alto, por lo que nos hace suponer que algunos de los individuos que se siguen conservando como positivos en el inter de 7 años es porque la presencia del vector se puede seguir dando en el peri domicilio donde habitan mamíferos de sangre caliente, que son reservorios del Trypanosoma cruzi y que pueden ser llevados nuevamente hacia el interior de las viviendas a través de la capacidad de volar que tienen casi todas las especies de insectos reduvidos transmisoras del parásito, o bien esa contínua reinfección pudo haber sido en el médio ambiente rural, ya que la mayoría de individuos investigados realizan actividades de ganadería o de agricultura y eso los obliga a dormir, en ocasiones, en el campo en donde con mayor facilidad pueden ser agredidos por estos artrópodos, vale la pena mencionar, que la mayoría de los individuos que siguen serológicos positivos continúan ante la presencia del vector.

Quizás, uno de los puntos más importantes de hallazgo en este estudio, fue la presencia de casos nuevos y de seroconversiones.
Con relación a los casos nuevos se puede explicar la aparición de los mismos debido a que los individuos estudiados tuvieron un período de tiempo de 7 años para poder estar en contacto con algún insecto reduvido y como fue explicado en los párrafos anteriores existen una gran cantidad de probabilidades que dicho contacto se haya dado. Al analizar la vivienda con riesgo $y$ los casos nuevos encontramos una $p<0,004$ y un odds ratio de 2,00 con límites de confianza que incluyen el $1 \mathrm{y}$ al analizarla con la presencia del vector el valor de $p$ aumentó a 0,0000001 con un odds ratio de 3,30 con límites de confianza que no incluyen el 1, lo cual nos demuestra que en la presencia de casas con riesgo existe la presencia del vector y esto hace que la aparición de casos nuevos se explique por sí misma.

En lo que respecta a las seroconversiones, 64 en total de los individuos estudiados, aunque no es un porcentaje muy alto $(2,3 \%)$ nos podría hacer pensar en primera instancia en una falla de la prueba serológica, lo cual nosotros descartamos, por lo que se describirá a continuación: el antígeno que se utilizó en las dos ocasiones fue un antígeno de procedencia Argentina elaborado con la misma cepa, denominada Tulawen, el de 1987 fue obsequiado por el Instituto Mario Fatala Chaven de Buenos Aires, Argentina y el de 1994 fue comprado a laboratorios Wiener, Argentina con el nombre comercial de Chaga-test, de esta manera se minimiza la posibilidad de cambios en los resultados serológicos por el uso de dos antígenos diferentes; las pruebas que se utilizaron fueron las mismas (hemaglutinación indirecta e inmunofluorescencia indirecta) lo que elimina también alguna alteración de los resultados. Se hizo un control de calidad interno, en el cual se usaron sueros controles positivos y negativos, algunos de éstos vienen dentro del equipo de Chaga-test y otros los usamos de nuestra seroteca provenientes de pacientes 
enfermos a los que se les aisló el parásito. Cuando existió alguna duda, en el procesamiento o en la lectura las pruebas, se repitieron hasta esclarecer esas dudas. También se llevó a cabo un control de calidad externo. Este control fue realizado por el laboratorio de referencia serológica de la Organización Mundial de Salud que se encuentra en la ciudad de Goiania en el Estado de Goiás en Brasil; este laboratorio es dirigido por el Dr. Alejandro Luquetti. A este laboratorio de referencia se trasladaron en las dos ocasiones ( 87 y 94 ) sueros de, aproximadamente, el $10 \%$ de los individuos estudiados por nosotros tanto positivos como negativos, para tener mayor confiabilidad en los resultados serológicos y aceptar la presencia de los seroconvertidos. Existía preocupación por la presencia de las seroconversiones, pero, encontramos algunas publicaciones que relatan una situación parecida pero todavía más inverosímil. El Dr. Luquetti encontró seronegativización en pacientes enfermos, a los que se les había aislado el Trypanosoma cruzi ${ }^{56}$, hallazgo que también sucedió en Costa Rica con Zeledón ${ }^{12}$. Esto nos demuestra que la enfermedad de Chagas puede tener comportamientos bastante raros en lo que a la respuesta inmune se refiere y esto es dado desde su descubrimiento, ya que la niña Berenice, de 9 años, en quién se encontró por primera vez el parásito en 1911, murió por una causa diferente a Chagas.

Al hacer el análisis estadístico de las 64 seroconversiones $(2,3 \%)$ de los casos estudiados pudimos observar que existe una significancia estadística con $p<0.05$ al cruce con las variables vivienda con riesgo y presencia del vector, lo que nos permite mencionar que el hecho de no convivir con vectores y de mejorar las viviendas (situación misma que hace que los vectores se destierren) puede permitir que los individuos tengan seroconversiones. Como comentario final, nosotros pensamos que las condiciones en que viven los individuos de las localidades rurales del Estado de Jalisco favorecen a que las infecciones continúen.

\section{AGRADECIMIENTOS}

Los autores agradecen la colaboración del Dr. Alejandro Luquetti Jefe del laboratorio de referencia para diagnóstico serológico de la OMS, de la ciudad de Goiania en el Estado de Goiás, Brasil, por el control de calidad externo que nos brindó.

\section{REFERENCIAS BIBLIOGRAFICAS}

1. Castro C, Rezende JM, Camargo M, Cerisola A, Prata A, Macedo V. Esofagopatia chagasica no municipio de Mambaí. Revista da Sociedade Brasileira de Medicina Tropical 17 (supl):22, 1984.

2. Cuartero LM, Ponce D, Recco R. Cinco nuevos casos de enfermedad de Chagas en Zacatecas y Jalisco, en la República Mexicana. Revista de Investigación de Salud Pública 27: 29-36, 1967.

3. Hernandez M, Ericson C, Delgadillo C. New focus of chagas disease in Mexico. Lancet 100:23, 1987.

4. Instituto Nacional de Diagnostico e Investigacion de la Enfermedad de Chagas "Dr. Mario Fatala Chaben". Subsecretaría de Programas de Salud. Ministerio de Salud y Acción Social. Manual sobre Enfermedad de Chagas, 1985.

5. Luquetti AO. Megaesôfago e anticorpos antiTrypanosoma cruzi. Revista Goiana de Medicina 33:116, 1987.

6. Luquetti AO. Use of Trypanosoma cruzi defined proteins for diagnosis multicentre trial. Serological and technical aspects. Memórias do Instituto Oswaldo Cruz 85:497505, 1990.
7. Mazzoti L. Dos casos de enfermedad de Chagas en el Estado de Oaxaca, México. Gaceta Médica Mexicana 70:417-420, 1940.

8. Prata A. Significance of Trypanosoma cruzi diferentiation and selection, relationship whit clinical and epidemiological varieties. Revista da Sociedade Brasileira de Medicina Tropical 18(supl):9-16, 1985.

9. Publicacion Técnica del Instituto Nacional de Diagnostico y Referencia Epidemiológica no 8. "La enfermedad de Chagas". Dirección General de Epidemiología. Secretaría de Salud. México DF, 1991.

10. Trujillo Contreras F, Martínez LC, Hernández R, Lozano F, Ruvalcaba S. Estudio epidemiólogico de la prevalencia de la enfermedad de Chagas en el estado de Jalisco, México, (reporte preliminar). Revista de la Asociación Médica de Jalisco. Nueva Epoca 3:28-31, 1987.

11. Wiener laboratorios. Chagatest HAI. Manual de procedimientos. Rosario Argentina, 1993.

12. Zeledon R, Dias JC, Brilla Salazar A, Resende JM, Vargas LG, Urbina A. In: Resumo da II Reunião Anual de Pesquisa Aplicada de Chagas, Araxá, p. 18, 1985. 